\title{
Inverse problem for the identification of a memory kernel from Maxwell's system integro - differential equations for a homogeneous anisotropic media
}

\author{
D. K. Durdiev \\ Bukhara State University, \\ Bukhara, Uzbekistan \\ durdiev65@mail.ru
}

PACS 02.30.Zz

DOI 10.17586/2220-8054-2015-6-2-268-273

We consider the problem of reconstructing the time-dependent history of electromagnetic fields from Maxwell's system of equations for an homogeneous anisotropic medium. As additional information, the Fourier image of electric and magnetic field intensity vectors for values $\nu=0$ of transformation parameter are given. It is shown that if the given functions satisfy some conditions of agreement and smoothness, the solution of the posed problem is uniquely defined in a class of continuously differentiable functions.

Keywords: inverse problem, integro-differential equation, delta function, Fourier transformation, agreement condition.

Received: 12 January 2015

Revised: 16 January 2015

\section{Setting up the problem and main result}

The unique dependence of $D$ and $B$ (the electric displacement and the magnetic induction, respectively) on $E$ and $H$ (the intensities of the respective fields) at the same instance of time is violated in rapidly varying electromagnetic fields whose frequencies are not small compared with the electric and magnetic polarization onset frequencies typical for the medium. The values of $D$ and $B$ at a given time have been proven to not only depend on $E$ and $H$, but also on the entire time history of these fields (such a medium is called a medium with aftereffect) [1]:

$$
\begin{gathered}
D(x, t)=\hat{\epsilon} E+\int_{0}^{t} \varphi(t-\tau) E(x, t) d \tau, \\
B(x, t)=\hat{\mu} H+\int_{0}^{t} \psi(t-\tau) H(x, t) d \tau, \\
E=\left(E_{1}, E_{2}, E_{3}\right), H=\left(H_{1}, H_{2}, H_{3}\right), D=\left(D_{1}, D_{2}, D_{3}\right), \\
B=\left(B_{1}, B_{1}, B_{3}\right), x=\left(x_{1}, x_{2}, x_{3}\right),
\end{gathered}
$$

where $\hat{\epsilon}=\left(\hat{\epsilon}_{i j}\right)_{3 \times 3}$ and $\hat{\mu}=\left(\hat{\mu}_{i j}\right)_{3 \times 3}$ are the permittivity and permeability matrices, respectively; $\varphi(t)=\operatorname{diag}\left(\varphi_{1}, \varphi_{2}, \varphi_{3}\right)$ and $\psi(t)=\operatorname{diag}\left(\psi_{1}, \psi_{2}, \psi_{3}\right)$ are diagonal matrices representing the memory. 
Many technically important materials and crystals which have become popular in new technologies are anisotropic. The physical properties of homogeneous isotropic crystals do not depend on the direction and the position inside the medium. At the same time, physical properties of anisotropic crystals essentially depend on orientation and position. An anisotropic crystal is called homogeneous when its physical properties depend on orientation and do not depend on position.

Suppose that, according to (1), the vectors $E$ and $H$ form a solution of the Cauchy problem for the system of Maxwell's equations for homogeneous anisotropic crystals with zero initial data:

$$
\begin{gathered}
\nabla \times H=\frac{\partial D(x, t)}{\partial t}+j, \quad \nabla \times E=-\frac{\partial B(x, t)}{\partial t}, \quad(x, t) \in \mathbb{R}^{4}, \\
\left.E\right|_{t \leq 0}=0,\left.\quad H\right|_{t \leq 0}=0 .
\end{gathered}
$$

where $j=\left(j_{1}, j_{2}, j_{3}\right)$ is the density of the electric current with components $j_{i}=j_{i}(x, t)$. The matrices $\hat{\epsilon}$ and $\hat{\mu}$ in equations (1) are assumed to be known constant matrices. Moreover, $\hat{\epsilon}-$ is a symmetric positive definite matrix. We will solve problems (1) and (2) for the case in which the function $j(x, t)$ has the form:

$$
j(x, t)=\vec{e} \delta(x) \delta(t)
$$

where $\vec{e}=(1,0,0)$ is unit vector; $\delta(x)=\delta\left(x_{1}\right) \delta\left(x_{2}\right) \delta\left(x_{3}\right)$ is the Dirac delta function of the space variable concentrated at $x_{1}=0, x_{2}=0, x_{3}=0 ; \delta(t)$ is the Dirac delta function of the time variable concentrated at $t=0$.

The problem in which the vectors $E(x, t), H(x, t)$ should be determined from (1) (3) for a given matrix functions $\varphi(t), \psi(t)$ will be called the direct problem.

Let $\tilde{E}=\left(\tilde{E}_{1}, \tilde{E}_{2}, \tilde{E}_{3}\right)(\nu, t), \tilde{H}=\left(\tilde{H}_{1}, \tilde{H}_{2}, \tilde{H}_{3}\right)(\nu, t)$ be the Fourier image of $E(x, t)$, $H(x, t)$ with respect to $x=\left(x_{1}, x_{2}, x_{3}\right) \in \mathbb{R}^{3}$, respectively, i.e.:

$$
\begin{gathered}
\tilde{E}_{j}(\nu, t)=\int_{R^{3}} E_{j}(x, t) e^{i(x, \nu)} d x, \quad \tilde{H}_{j}(\nu, t)=\int_{R^{3}} H_{j}(x, t) e^{i(x, \nu)} d x, \\
\nu=\left(\nu_{1}, \nu_{2}, \nu_{3}\right) \in \mathbb{R}^{3}, \quad(x, \nu)=\sum_{\lambda=1}^{3} x_{\lambda} \nu_{\lambda}, \quad j=1,2,3 .
\end{gathered}
$$

We pose the following inverse problem: find the functions $\varphi(t)=\operatorname{diag}\left(\varphi_{1}, \varphi_{2}, \varphi_{3}\right)$, $\psi(t)=\operatorname{diag}\left(\psi_{1}, \psi_{2}, \psi_{3}\right)$ occurring in the integral in equations (1) from the information on the Fourier image $\tilde{E}, \tilde{H}$ of the electric and magnetic fields at an arbitrary time $t \geq 0$ for the values $\nu=0$ of the Fourier transformation:

$$
\left(\tilde{E}_{1}, \tilde{E}_{2}, \tilde{E}_{3}, \tilde{H}_{1}, \tilde{H}_{2}, \tilde{H}_{3}\right)(0, t)=g(t), \quad g(t)=\left(g_{1}, g_{2}, \ldots, g_{6}\right) .
$$

Definition. Solutions of the inverse problem are matrix functions $\varphi(t)$ and $\psi(t)$, such that the corresponding solution of problem (1) -(3) satisfies condition (4).

Among the problems devoted to determining a sub-integral function, belonging to hyperbolic equations, we note works [2,3]. In work [2], the problem of determining the memory, belonging to a three-dimensional wave equation with delta function at the right side is investigated. Furthermore, in work [3], this problem is generalized in the case of hyperbolic equation of the second order with constant main part and variable coefficients at minor derivatives. Similar problems with distributed sources of disturbance are seen in 
works [4,5]. In article [6], the problem of reconstructing the time history of an electric field from the electrodynamic equation is investigated. Also, we recall that papers $[7,8,9,10]$ are concerned with the determination of memory kernel from integro-differential equations with an integral of convolution type. In the present paper, we consider the problem of reconstructing the time-dependent history of the electromagnetic fields from Maxwell's system of equations for an anisotropic medium. It is shown that if the vector function $g(t)$ satisfies some conditions of agreement and smoothness, the solution to the inverse problem is uniquely defined in a class of continuously differentiable functions on the intercept $[0, T]$, where $T$ is any positive fixed number.

The main result of the present paper is the following theorem.

Theorem. Suppose that $g(t) \in C^{2}[0, T], g(0) \neq 0$ and the agreement conditions:

$$
\sum_{k=1}^{3} \hat{\epsilon}_{1 k} g_{k}(0)=-1, \quad \sum_{k=1}^{3} \hat{\epsilon}_{i k} g_{k}(0)=0, \quad i=2,3 ; \quad \sum_{k=1}^{3} \hat{\mu}_{j k} g_{3+k}(0)=0, \quad j=1,2,3
$$

hold. Then, the inverse problem (1) -(4) has a unique solution $(\varphi(t), \psi(t)) \in \mathbb{C}^{1}[0, T]$ for any fixed $T>0$.

\section{Maxwell's equations as a first order symmetric hyperbolic system} system:

Equations (1) -(3) can be written as the following first order symmetric hyperbolic

$$
A_{0} \frac{\partial V}{\partial t}+\sum_{i=0}^{3} A_{i} \frac{\partial V}{\partial x_{i}}+\Phi_{0} V+\int_{0}^{t} \Phi^{\prime}(t-\tau) V(x, \tau) d \tau=F(x, t)
$$

with the initial condition:

where:

$$
\left.V\right|_{t \leq 0}=0
$$

$$
\begin{gathered}
A_{0}:=\left(\begin{array}{cc}
\hat{\epsilon} & 0 \\
0 & \hat{\mu}
\end{array}\right)_{6 \times 6}, \quad A_{j}:=\left(\begin{array}{cc}
0 & A_{j}^{1} \\
\left(A_{j}^{1}\right)^{*} & 0
\end{array}\right)_{6 \times 6}, \quad A_{1}^{1}:=\left(\begin{array}{ccc}
0 & 0 & 0 \\
0 & 0 & 1 \\
0 & -1 & 0
\end{array}\right), \\
A_{2}^{1}:=\left(\begin{array}{ccc}
0 & 0 & -1 \\
0 & 0 & 0 \\
1 & 0 & 0
\end{array}\right), \quad A_{3}^{1}:=\left(\begin{array}{ccc}
0 & 1 & 0 \\
-1 & 0 & 1 \\
0 & 0 & 0
\end{array}\right), \quad \Phi_{0}:=\left(\begin{array}{cc}
\varphi(0) & 0 \\
0 & \psi(0)
\end{array}\right)_{6 \times 6}, \\
\Phi^{\prime}(t):=\left(\begin{array}{cc}
\varphi^{\prime}(t) & 0 \\
0 & \psi^{\prime}(t)
\end{array}\right)_{6 \times 6}, \quad V:=(E, H)^{*}, \quad F:=\left(-j, 0_{1 \times 3}\right)^{*} ;
\end{gathered}
$$

* is the symbol of transposition; $0_{1 \times 3}$ denote the vector line with elements $0,0,0$; $\Phi^{\prime}(t):=(\partial / \partial t) \Phi(t)$.

We apply to both parts of (5) and (6) the Fourier transformation. The Fourier transform of the vector function $V(x, t)$ exists at any finite $t$, since the vector function $V(x, t)$ as the solution of the direct problem (5) and (6) is a sum of a certain singular generalized vector function and a regular vector function, the support of the vector function $V(x, t)$ being finite [11, chapter 4]. For any fixed $\nu$, the vector function $\tilde{V}(\nu, t)((\tilde{V}(\nu, t)-$ the Fourier transformation of $V(x, t)$ with respect to $x)$ satisfies differential equation: 


$$
A_{0} \frac{\partial \tilde{V}}{\partial t}-i B \tilde{V}+\int_{0}^{t} \Phi^{\prime}(t-\tau) \tilde{V}(\nu, \tau) d \tau=\tilde{F}(t),
$$

the initial, and as follows from (4), the supplementary conditions, respectively:

$$
\begin{gathered}
\left.\tilde{V}\right|_{t \leq 0}=0, \\
\left.\tilde{V}\right|_{\nu=0}=g(t), \quad g(t)=\left(g_{1}, g_{2}, \ldots, g_{6}\right), \quad t \geq 0 .
\end{gathered}
$$

In equation (7), we denoted $B:=\sum_{j=1}^{3} \nu_{j} A_{j}+\Phi_{0}, \tilde{F}(t)=-\overrightarrow{e_{0}} \delta(t), \overrightarrow{e_{0}}:=(1,0,0,0,0,0)^{*}$.

We compute $A_{0}^{-1}$, which is the inverse to $A_{0}$. If we denote by $\epsilon=\left(\epsilon_{i j}\right), \mu=\left(\mu_{i j}\right)$ the matrices which are the inverse to $\hat{\epsilon}, \hat{\mu}$, respectively, then:

$$
A_{0}^{-1}=\left(\begin{array}{cc}
\epsilon & 0 \\
0 & \mu
\end{array}\right)_{6 \times 6} .
$$

When we multiply the left-hand side of (7) by $A_{0}^{-1}$, we get:

$$
I \frac{\partial \tilde{V}}{\partial t}-i C \tilde{V}+\int_{0}^{t} \Psi(t-\tau) \tilde{V}(\nu, \tau) d \tau=F_{0}
$$

where $I$ is the identity matrix,

$$
C:=\left(\begin{array}{cc}
\epsilon \varphi(0) & \epsilon \Sigma_{i=0}^{3} \nu_{i} A_{i}^{1} \\
\mu \Sigma_{i=0}^{3} \nu_{i}\left(A_{i}^{1}\right)^{*} & \mu \psi(0)
\end{array}\right)_{6 \times 6},
$$

$\Psi(t):=A_{0}^{-1} \Phi^{\prime}(t), F_{0}:=A_{0}^{-1} \tilde{F}=-A_{0}^{-1} \overrightarrow{e_{0}} \delta(t)$.

Thus, inverse problems (1) - (4) are reduced to the problem of determining the kernel $\Psi(t)$ of the integral part in equation (10) on the bases of equalities (8) - (10).

\section{Proof of the main result}

We integrate the differential equation (10). Using the initial condition (8), one gets:

$$
\tilde{V}(\lambda, t)=-A_{0}^{-1} \overrightarrow{e_{0}}+i C \int_{0}^{t} \tilde{V}(\lambda, \tau) d \tau-\int_{0}^{t} \int_{0}^{\tau} \Psi(\alpha) \tilde{V}(\lambda, \tau-\alpha) d \alpha d \tau .
$$

Taking into account (9), from the equality (11) we obtain:

$$
g(t)=-A_{0}^{-1} \overrightarrow{e_{0}}+i C_{0} \int_{0}^{t} g(\tau) d \tau+\int_{0}^{t} \Psi(\alpha) \int_{\alpha}^{t} g(\tau-\alpha) d \tau d \alpha, \quad t>0,
$$

where $C_{0}:=A_{0}^{-1} \Phi_{0}$. By differentiating the equation (12), we derive:

$$
\int_{0}^{t} \Psi(\tau) g(t-\tau) d \tau=g^{\prime}(t)-i C_{0} g(t)
$$

In the equality, assuming $t=0$ and taking into account $C_{0}=A_{0}^{-1} \Phi_{0}$, we get:

$$
g^{\prime}=i A_{0}^{-1} \Phi_{0} g(0)
$$


It follows from the last equation that the elements of the matrix $\Phi_{0}$ are expressed by the known numbers:

$$
\varphi_{i}(0)=-\frac{i}{g_{i}(0)} \sum_{k=1}^{3} \hat{\epsilon}_{i k} g_{k}^{\prime}(0) ; \quad \psi_{i}(0)=-\frac{i}{g_{3+i}(0)} \sum_{k=1}^{3} \hat{\mu}_{i k} g_{3+k}^{\prime}(0), \quad i=1,2,3 .
$$

Furthermore, constants $\varphi_{i}(0), \psi_{i}(0), i=1,2,3$ will be assumed as knowns.

From (13), by differentiating, we obtain:

$$
\Psi(t) g(0)+\int_{0}^{t} \Psi(\tau) g^{\prime}(t-\tau) d \tau=g^{\prime \prime}(t)-i A_{0}^{-1} g^{\prime}(t)
$$

When we multiply the left-hand part of the last equality by $A_{0}$, we find:

$$
\Phi^{\prime}(t) g(0)+\int_{0}^{t} \Phi^{\prime}(\tau) g^{\prime}(t-\tau) d \tau=A_{0} g^{\prime \prime}(t)-i \Phi_{0} g^{\prime}(t) .
$$

The last equality is the linear integral second-order equation of Volterra type with respect to the matrix function $\Phi^{\prime}(t)$. It can be written relative to the components of matrix $\Phi^{\prime}(t)$. They are as follows:

$$
\begin{gathered}
\varphi_{j}^{\prime}(t)+\int_{0}^{t} \varphi_{j}^{\prime}(\tau) \frac{g_{j}^{\prime}(t-\tau)}{g_{j}(0)} d \tau=\frac{1}{g_{j}(0)}\left\{\sum_{k=1}^{3} \hat{\epsilon_{j k}} g_{k}^{\prime \prime}-i \varphi_{j}(0) g_{k}^{\prime}(t)\right\}, \quad j=1,2,3 ; \\
\psi_{j}^{\prime}(t)+\int_{0}^{t} \psi_{j}^{\prime}(\tau) \frac{g_{3+j}^{\prime}(t-\tau)}{g_{3+j}(0)} d \tau=\frac{1}{g_{3+j}(0)}\left\{\sum_{k=1}^{3} \hat{\mu_{j k} g_{3+k}^{\prime \prime}}(t)-i \psi_{j}(0) g_{3+k}^{\prime}(t)\right\}, \quad j=1,2,3 .
\end{gathered}
$$

To integral equations (14) and (15), we combine the following obvious relations:

$$
\varphi_{j}(t)-\int_{0}^{t} \varphi_{j}^{\prime}(\tau) d \tau=\varphi_{j}(0), \quad \psi_{j}(t)-\int_{0}^{t} \psi_{j}^{\prime}(\tau) d \tau=\psi_{j}(0), \quad j=1,2,3 .
$$

Equations (14) - (16) are the linear integral second-order equations of the Volterra type with respect to unknown functions $\varphi_{i}^{\prime}, \varphi_{i}, \psi_{i}^{\prime}, \psi_{i}, i=1,2,3$. As known, these equations have unique solutions.

\section{References}

[1] Landau L. D. and Lifshits E. M. Continuum Electrodynamics. Nauka, Moscow, 1959. (in Russian)

[2] Durdiev D. K. An inverse problem for a three-dimensional wave equation in the medium with memory. Math. Anal. and Disc. Math., Novosibirsk, NGU, P. 19-26 (1989). (in Russian)

[3] Durdiev D. K. To the question of correctness of one inverse problem for hyperbolic integro-differential equation. Sib. Math. Journ., 33 (3), P. 69-77 (1992). (in Russian)

[4] Lorensi A. An identification problem related to a nonlinear hyperbolic integro-differential equation. Nonlinear Analysis: Theory, Methods and Applications, 22, P. 297-321 (1994).

[5] Janno J. and Von Welfersdorf L. Inverse problems for identification of memory kernels in viscoelasticity. Math. Methods in Appl. Sciences, 20 (4), P. 291-314 (1997). 
[6] Durdiev D. K. Global solvability of an inverse problem for an integro-differential equation of electrodynamics. Differential Equations, (44) (7), P. 893-899 (2008).

[7] Jaan J. and Von Wolfersdorf L. An inverse problem for identification of a time- and space-dependent memory kernel in viscoelasticity. Inverse Problems, 17, P. 13-24 (2001).

[8] Colombo F. and Guidetti D. A global in time existence and uniqueness result for a semilinear integrodifferential parabolic inverse problem in Sobolev spaces. Math. Methods in Appl. Sciences, 17, P. 1-29 (2007).

[9] Colombo F. and Guidetti D. Some results on the Identification of memory kernels. Oper. Theory: Adv. Appl., 216, P. 121-138 (2011).

[10] Favaron A. Identification of Memory Kernels Depending on Time and on an Angular Variable. Zeitschrift fur Analysis und ihre Anwendungen, 24, (4), P. 735-762 (2005).

[11] Romanov V. G. Inverse problems of mathematical physics. Nauka, Moscow, 1984. (in Russian) 